\title{
Simultaneous Assessment of Hepatic Transport and Metabolism Pathways with a Single Probe Using Individualized PBPK Modeling of ${ }^{14} \mathrm{CO}_{2}$ Production Rate Data ${ }^{[}$
}

\author{
Yoko Franchetti and (1)Thomas D. Nolin
}

\begin{abstract}
Departments of Pharmaceutical Sciences (Y.F.) and Pharmacy and Therapeutics (T.D.N.), Center of Clinical Pharmaceutical Sciences, University of Pittsburgh School of Pharmacy, Pittsburgh, Pennsylvania
\end{abstract}

Received February 21, 2019; accepted July 31, 2019

\begin{abstract}
Erythromycin is a substrate of cytochrome P4503A4 (CYP3A4) and multiple drug transporters. Although clinical evidence suggests that uptake transport is likely to play a dominant role in erythromycin's disposition, the relative contributions of individual pathways are unclear. Phenotypic evaluation of multiple pathways generally requires a probe drug cocktail. This approach can result in ambiguous conclusions due to imprecision stemming from overlapping specificity of multiple drugs. We hypothesized that an individualized physiologically based pharmacokinetic modeling approach incorporating ${ }^{14} \mathrm{CO}_{2}$ production rates (iPBPK-R) of the erythromycin breath test (ERMBT) would enable us to differentiate the contribution of metabolic and transporter pathways to erythromycin disposition. A sevencompartmental physiologically based pharmacokinetic (PBPK) model was built for ${ }^{14} \mathrm{C}$-erythromycin administered intravenously. Transporter clearance and CYP3A4 clearance were embedded in hepatic compartments. ${ }^{14} \mathrm{CO}_{2}$ production rates were simulated taking the first derivative of by-product ${ }^{14} \mathrm{CO}_{2}$ concentrations. Parameters related to nonrenal elimination pathways were estimated by model fitting the ERMBT data of 12 healthy subjects individually. Optimized iPBPK-R models fit the individual rate data well. Using one probe, nine PBPK parameters were simultaneously estimated per individual. Maximum velocity of uptake transport, CYP3A4 clearance, total passive diffusion, and others were found to collectively control ${ }^{14} \mathrm{CO}_{2}$ production
\end{abstract}

rates. The median CYP3A4 clearance was $12.2 \%$ of the input clearance. Male subjects had lower CYP3A4 activity than female subjects by $11.3 \%$. We applied iPBPK-R to ERMBT data to distinguish and simultaneously estimate the activity of multiple nonrenal elimination pathways in healthy subjects. The iPBPK-R framework is a novel tool for delineating rate-limiting and nonrate-limiting elimination pathways using a single probe.

\section{SIGNIFICANCE STATEMENT}

Our developed individualized physiologically based pharmacokinetic modeling approach incorporating rate data (iPBPK-R) enabled us to distinguish and simultaneously estimate the activity of multiple nonrenal elimination pathways of erythromycin in healthy subjects. A new interpretation of erythromycin breath test (ERMBT) data was also obtained via iPBPK-R. We found that rate data have rich information allowing estimation of per-person PBPK parameters. This study serves as proof of principle that the iPBPK-R framework is a novel tool for delineating rate-limiting and non-rate-limiting elimination pathways using a single probe. iPBPK-R can be applied to other rate-derived data beyond ERMBT. Potential areas of application include drug-drug interaction, pathophysiological effects on drug disposition, and the role of biomarkers on hemodialysis efficiency utilizing estimated adjustment factors with correlation analysis.
This work is an extension of a study previously funded by the Satellite Healthcare Research Foundation. Y.F. is supported by an appointment to the Research Participation Program at the Center for Drug Evaluation and Research, administered by the Oak Ridge Institute for Science and Education through an interagency agreement between the U.S. Department of Energy and the U.S. Food and Drug Administration. T.D.N. is supported in part by the National Institutes of Health National Institute of General Medical Sciences [Grant R01-GM107122]. This work used the Extreme Science and Engineering Discovery Environment (XSEDE), which is supported by National Science Foundation grant number ACI-1548562, and XSEDE Bridges at the Pittsburgh Supercomputing Center through allocation BIO180015.

https://doi.org/10.1124/jpet.119.257212.

S This article has supplemental material available at jpet.aspetjournals.org.

\section{Introduction}

The macrolide antibiotic erythromycin is a nonrenally cleared drug that undergoes hepatic elimination via multiple pathways (Lam et al., 2006; Nolin, 2008; Benet, 2009; Franke et al., 2011; Chu et al., 2013). In the past, erythromycin was commonly used as a metabolic probe drug. It was administered intravenously and employed in the erythromycin breath test (ERMBT) to noninvasively measure in vivo hepatic CYP3A4 activity (Turgeon et al., 1994; Hirth et al., 2000; Nolin et al., 2006a; de Wildt et al., 2007). Use of the ERMBT in this manner relied on the premise that intravenously administered radiolabeled erythromycin undergoes N-demethylation by CYP3A4

ABBREVIATIONS: AUC, area under the curve; BUN, blood urea nitrogen; ERMBT, erythromycin breath test; ES, extracellular space; iPBPK-R, individualized physiologically based pharmacokinetic modeling approach incorporating rate data; IVIVE, in vitro-in vivo extrapolation; LC, liver cell; MRP, multidrug resistance-associated protein; OATP, organic anion transporting peptide; ODE, ordinary differential equation; P-gp, P-glycoprotein; PBPK, physiologically based pharmacokinetic; PK, pharmacokinetic. 
in the liver and that ${ }^{14} \mathrm{CO}_{2}$, a final by-product of the CYP3A4mediated elimination pathway, is rapidly detected in breath (Nolin et al., 2006a; de Wildt et al., 2007). However, it is now known that erythromycin also undergoes hepatic drug transport-that is, hepatic clearance by organic anion transporting polypeptides (OATPs), P-glycoprotein (P-gp), and multidrug resistance-associated protein 2 (MRP2)-mediated elimination pathways (Lan et al., 2000; Sun et al., 2004; Kurnik et al., 2006; Lam et al., 2006; Frassetto et al., 2007; Franke et al., 2011; Chu et al., 2013).

After hepatic uptake transport by OATPs into the cells, ${ }^{14} \mathrm{C}$-erythromycin partly undergoes $\mathrm{N}$-demethylation by CYP3A4 and gets converted to N-demethylated metabolites and ${ }^{14} \mathrm{C}$-formaldehyde. ${ }^{14} \mathrm{C}$-formaldehyde gets mostly converted to ${ }^{14} \mathrm{C}$-formate. Subsequently, ${ }^{14} \mathrm{CO}_{2}$ is released and exhaled in breath in this CYP3A4-mediated pathway. Meanwhile, unmetabolized drug and $\mathrm{N}$-demethylated metabolites are excreted by the transporter P-gp (Kurnik et al., 2006; Frassetto et al., 2007). Accordingly, interpretations of ERMBT data were misleading under the assumption that the erythromycin clearance was dependent solely on CYP3A4-mediated metabolism (Rivory and Watkins, 2001; Rivory et al., 2001). Although some studies with ERMBT concluded that there was a positive or inverse relationship between an ERMBT measure and a change in CYP3A4 activity (Turgeon et al., 1994; Hirth et al., 2000; Nolin et al., 2006a), a pilot pediatric study showed neither a consistent peak of ${ }^{13} \mathrm{CO}_{2}$ flux across preterm infants nor a potential use of ERMBT for measuring CYP3A activity in these patients (de Wildt et al., 2007). The ERMBT has been reported to correlate poorly with the clearance of other drugs (Krivoruk et al., 1994; Krecic-Shepard et al., 1999). Furthermore, clinical studies with OATP and/or P-gp inhibitors (Kurnik et al., 2006; Frassetto et al., 2007) or a MRP2 variant (Franke et al., 2011) indicated that the effects of uptake and efflux transporters on erythromycin disposition would need to be accounted for in the interpretation of ERMBT results. Consequently, research using the ERMBT became less attractive and less common. In fact, it was determined that more research on the ERMBT itself was necessary to establish its reliability (Watkins, 1994; Spivey, 2000; Rivory et al., 2001; Frassetto et al., 2007).

The ${ }^{14} \mathrm{CO}_{2}$ production rate measurement of ERMBT results may provide a unique opportunity to differentiate contributions of metabolic versus transporter-mediated pathways to erythromycin disposition, since ${ }^{14} \mathrm{CO}_{2}$ concentration in breath is proportional to the ${ }^{14} \mathrm{CO}_{2}$ production rate in the hepatocyte. Moreover, contributions of specific pathways may be estimated within individuals or per person when physiologically based pharmacokinetic (PBPK) modeling is applied to ${ }^{14} \mathrm{CO}_{2}$ production rate data. By measuring the breath concentration as proxy for ${ }^{14} \mathrm{CO}_{2}$ production rate, a hidden behavioral signal can be captured, which is usually unrecognized and treated as noise in modeling mean drug concentrations (Oppenheim and Schafer, 2009). Thus, the advantage of incorporating
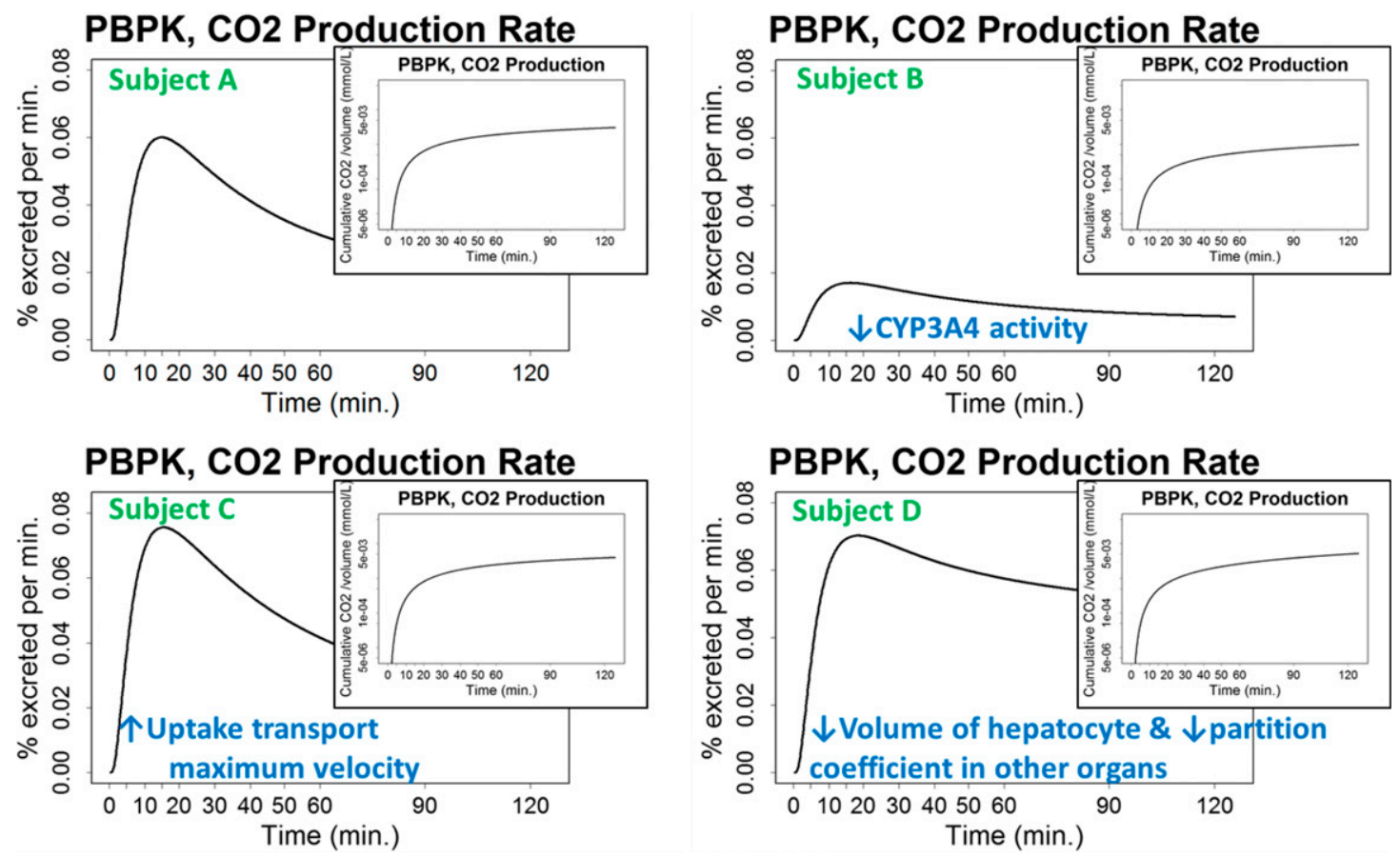

Fig. 1. Examples of the impact of $\mathrm{PBPK}$ parameters on the shape of the ${ }^{14} \mathrm{CO}_{2}$ production rate-time curves compared with the shape of the ${ }^{14} \mathrm{CO}_{2}$ concentration-time curves in the hepatocyte. In each of the four panels (subjects A-D), the main plot shows the ${ }^{14} \mathrm{CO}_{2}$ production rate-time curve and the inset smaller panel shows the corresponding ${ }^{14} \mathrm{CO}_{2}$ concentration-time curve. Both curves were simulated using PBPK modeling. The upper left panel illustrates the ${ }^{14} \mathrm{CO}_{2}$ production rate-time curve and ${ }^{14} \mathrm{CO}_{2}$ concentration-time curve for a hypothetical healthy subject denoted subject $\mathrm{A}$. In the upper right panel, subject $\mathrm{B}$ has a lower CYP3A4 clearance compared with the ${ }^{14} \mathrm{CO}_{2}$ production rate-time curve of subject $\mathrm{A}$ and the ${ }^{14} \mathrm{CO}_{2}$ production rate-time curve of subject $\mathrm{B}$ is flatter without presenting a sharp peak accordingly. In the lower left panel, subject $\mathrm{C}$ has a higher peak of the ${ }^{14} \mathrm{CO}_{2}$ production rate-time curve due to the increased maximum velocity of the uptake drug transport compared with subject A. In the lower right panel, subject D shows the greater AUC of the ${ }^{14} \mathrm{CO}_{2}$ production rate-time curve due to the decreased volume of the hepatocyte compared with subject $\mathrm{A}$. Subject $\mathrm{D}$ has less steep slopes in the first and second elimination phases of the ${ }^{14} \mathrm{CO}_{2}$ production rate-time curve due to the decreased partition coefficient of the combined other organs compared with those of subject A. Note that the curves of subjects B-D were simulated by changing particular parameter(s) based on the curves of subject A, and these subjects are hypothetical examples. 
measured ${ }^{14} \mathrm{CO}_{2}$ production rate into $\mathrm{PBPK}$ modeling is that the concentration change, or rate, contains greater information and is more sensitive to physiologic changes compared with concentration itself (Fig. 1). Therefore, multiple elimination pathways of a single drug can be differentially estimated using the rate data.

The aim of this work was to estimate the activity and corresponding contribution of multiple nonrenal clearance pathways to the elimination of a single probe (erythromycin) with our novel individualized physiologically based pharmacokinetic modeling approach using rate data (iPBPK-R). This study establishes proof of concept that rate data may be used as a method for estimating the activities and differentiating the contribution of multiple clearance pathways to the elimination of a single probe drug that exhibits overlapping substrate specificity. We anticipate that the iPBPK-R framework will be applicable to breath rate data of other compounds, such as volatile organic compounds (O'Hara et al., 2009; Grabowska-Polanowska et al., 2019), to evaluate drug-drug interactions, disease effects on drug disposition, and the relationship between potential biomarkers and intervention, among others.

\section{Materials and Methods}

Clinical Data Sources. The ${ }^{14} \mathrm{CO}_{2}$ production rate data were obtained from a prospective cohort study in which the ERMBT was administered to 12 healthy Caucasian subjects (seven male) (Nolin et al., 2006b). Briefly, a single $0.074 \mathrm{mmol}(0.04 \mathrm{mg}, 3 \mu \mathrm{Ci})$ dose of $\left[{ }^{14} \mathrm{C}-\mathrm{N}\right.$-methyl] erythromycin (Metabolic Solutions Inc., Nashua, NH) was intravenously administered and breath samples were collected immediately before receiving the dose and at 5, 10, 15, 20, 30, 40, 50, 60,90 , and 120 minutes postdosing as previously described (Nolin et al., 2006a). The study adhered to the Declaration of Helsinki and was approved by the Maine Medical Center Institutional Review
Board and the Radiation Safety Committee. Parameters for which no in vitro values were available in the published literature and that could not be calculated using the in vitro-in vivo extrapolation (IVIVE) approach were estimated by applying the PBPK-R model (described in the next subsections) to the previously published ${ }^{14} \mathrm{CO}_{2}$ production rate at 20 minutes (Frassetto et al., 2007).

PBPK Model Structure. As shown in Fig. 2, a PBPK model comprising six organ compartments was built with pharmacokinetic parameters for ${ }^{14} \mathrm{C}$-erythromycin. These compartments were artery (Art), Vein, lung (LG), liver (LV), kidney (KD), and combined other organs (OT). The liver compartment consisted of two subcompartments: extracellular space (ES) and liver cell (LC). A nonlinear meta-uptake transporter (OATPs are combined; see the Model Assumptions section) and total passive diffusion modeled the drug transfer between the ES and LC subcompartments. Nonlinear efflux transporters (P-gp and MRP2) and CYP3A4 clearance were modeled in the LC compartment. CYP3A4 clearance was observed to be linear, which is not surprising since a low dose of ${ }^{14} \mathrm{C}$-erythromycin was administered and it was expected that its concentration was well below the maximum velocity of nonlinear kinetics.

Ordinary Differential Equations, Kinetic Parameters, and Data Input. Changes in drug concentrations of all compartments and subcompartments contained within the PBPK model were mathematically described with a set of ordinary differential equations (ODEs) (see Supplemental Material). We used permeability-limited liver models in the ODEs associated with the ES and LC subcompartments (Jamei et al., 2014). In addition, we referred to Kanamitsu et al. (2000) for the metabolic by-product model to describe the series of non-ratelimiting steps from ${ }^{14} \mathrm{C}$-formaldehyde to bicarbonate and subsequent ${ }^{14} \mathrm{CO}_{2}$ generation within the LC subcompartment. This allowed us to approximate the $\mathrm{CO}_{2}$ concentration in the breath through the first derivative of the cumulative $\mathrm{CO}_{2}$ concentration generated in the $\mathrm{LC}$ subcompartment $\left(\mathrm{CO}_{2}\right.$ concentration rate of the $\mathrm{LC}$ subcompartment) (see Fig. 2 and the Model Assumptions section). Table 1 provides physiological and kinetic parameters that were calculated based on IVIVE. These parameter values were used as initial input values in the iPBPK-R model fitting.
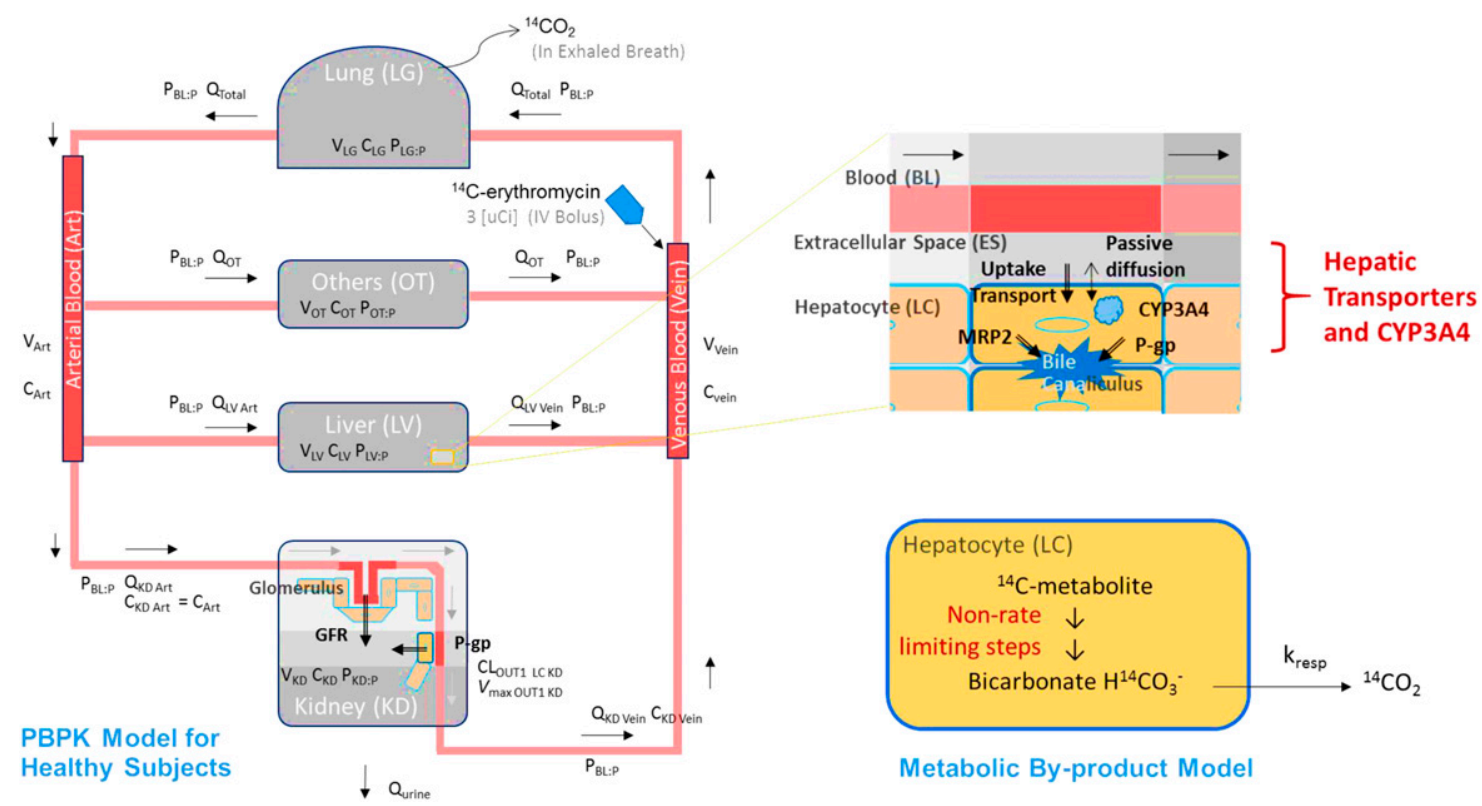

Fig. 2. Schematic representation of a PBPK model for describing the time profiles of ${ }^{14}$ C-erythromycin after intravenous ${ }^{14} \mathrm{C}$-erythromycin administration in a healthy subject. The PBPK model consisting of six organ compartments was built with pharmacokinetic parameters for ${ }^{14} \mathrm{C}$-erythromycin. The upper right panel shows the liver composed of two subcompartments (ES and LC) where nonlinear uptake and efflux transporters and linear enzymatic clearance were embedded. In the lower right panel, the metabolic by-product model is illustrated where the metabolite ${ }^{14} \mathrm{C}$-formate is converted to radiolabeled bicarbonate $\mathrm{H}^{14} \mathrm{CO}_{3}^{-}$in the CYP3A4-mediated pathway and dissolved in the LC subcompartment. The series of conversion from ${ }^{14} \mathrm{C}$-formaldehyde to $\mathrm{H}^{14} \mathrm{CO}_{3}^{-}$is assumed to be a rapid process and nonrate limiting. Then, ${ }^{14} \mathrm{CO}_{2}$ is produced and exhaled in breath as a result of the first-order elimination of bicarbonate (with a constant $k_{\text {resp }}$ ) from the LC subcompartment. IV, intravenous. 
TABLE 1

Parameters used for the erythromycin breath test simulations

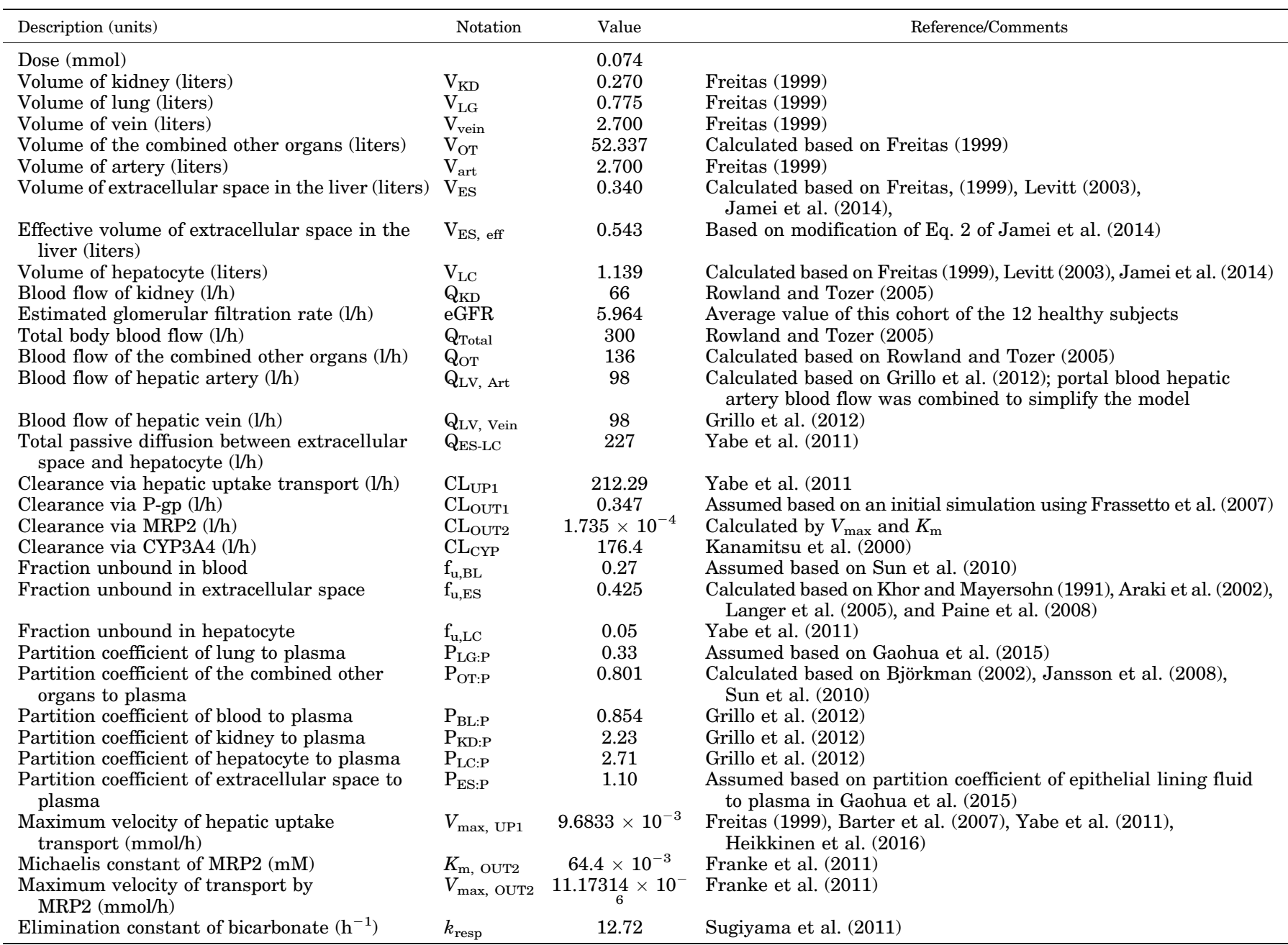

Rate Data and Reduced Order Model. Frequently sampled rate data has more information compared with equivalently sampled concentration data. However, it requires more effort to extract the information. Nonlinear model fitting of measured first derivative data is often performed to estimate sensitive parameters in established methodologies used in digital signal processing and other engineering disciplines (Oppenheim and Schafer, 2009). We modeled the ${ }^{14} \mathrm{CO}_{2}$ production rates from the ERMBT study using the same concept, which is illustrated in Fig. 1. Accordingly, the iPBPK-R method was applied to the ${ }^{14} \mathrm{CO}_{2}$ production rate data, and multiple hepatic elimination pathways (i.e., uptake and efflux transport and passive diffusion parameters) were estimated using the single probe drug ${ }^{14} \mathrm{C}$-erythromycin. To achieve high precision in model fitting to the high-resolution ${ }^{14} \mathrm{CO}_{2}$ rate data, we used a reduced order model (Schilders et al., 2008). Reduced order models are generally complex enough to capture the behavior of interest but simple enough so that the mathematical models are well-posed, providing estimable parameters. Based on this rationale, the seven-compartmental PBPK model was built as described in the above subsections PBPK Model Structure and Ordinary Differential Equations, Kinetic Parameters, and Data Input.

Model Assumptions. The number of compartments was limited to seven including subcompartments to facilitate fitting of PBPK models to ERMBT data while enabling estimation of the activity and corresponding of effect of CYP3A4 and drug transporters on erythromycin disposition. The modeling was carried out under the following assumptions:
- ${ }^{14} \mathrm{C}$-erythromycin is intravenously administered for 30 seconds.

- Except for the liver and kidney compartments, all compartments were assumed to be perfusion limited, where the distribution of a drug rapidly reaches equilibrium via passive diffusion and the unbound concentration in the compartment is the same as those in the diffused space at equilibrium. Perfusion-limited compartments were described with a well stirred model (Kanamitsu et al., 2000; Maeda and Sugiyama, 2013).

- Pathways from the ES compartment to the LC compartment for erythromycin are composed of total passive diffusion plus summation of uptake transporters only. Erythromycin mainly undergoes hepatic uptake transport by OATP isoforms OATP1B1 and OATP1B3. These uptake transport processes were modeled as a single meta-uptake process.

- $\mathrm{CO}_{2}$ is present in the systemic circulation under steady-state conditions. Accordingly, ${ }^{14} \mathrm{CO}_{2}$ is instantaneously released in breath via the lung without being stored in or released from any other compartments after it gets generated in the LC subcompartment.

- We assume that a metabolite ${ }^{14} \mathrm{C}$-formate is converted to radiolabeled bicarbonate $\mathrm{H}^{14} \mathrm{CO}_{3}$ - as a by-product in the CYP3A4-mediated pathway and dissolved in the LC subcompartment. The series of conversion from ${ }^{14} \mathrm{C}$-formaldehyde to ${ }^{14} \mathrm{C}$-formate through to $\mathrm{H}^{14} \mathrm{CO}_{3}$ - is rapid and not rate limiting as assumed by Sugiyama et al. (2011). The final by-product ${ }^{14} \mathrm{CO}_{2}$ in this elimination pathway is produced and exhaled in breath as a result of a first-order elimination of bicarbonate 
(with a constant $k_{\text {resp }}$ ) from the LC subcompartment (Sugiyama et al., 2011).

- The liver (composed of ES and LC subcompartments) and the kidneys (kidney compartment) are elimination organs. In addition, a parameter $\mu$ was included in the combined other organs compartment to explain the exponential decay that was seen in the observed ${ }^{14} \mathrm{CO}_{2}$ production rate data. We anticipated that this parameter $\mu$ would improve the model fitting. Thus, the exponential decay was assumed to explain any loss or decomposition of the drug before the drug reaches the liver in the system.

- The unbound drug, but not the bound drug, is subject to uptake and efflux transport, enzymatic metabolism, and elimination in the liver.

- For the intrinsic clearance of CYP3A, linearity was assumed after investigating the model fitting in comparison with nonlinear intrinsic clearance.

Simulations, Optimizations, and Sensitivity Analysis. We developed the individualized PBPK modeling of rate (iPBPK-R) framework shown in Fig. 3. Specifically, concentration-time curves of ${ }^{14} \mathrm{C}$-erythromycin and enzymatic by-products $\mathrm{H}^{14} \mathrm{CO}_{3}^{-}$and ${ }^{14} \mathrm{CO}_{2}$ were simulated for individual subjects based on the ODEs (Fig. 2; Supplemental Material). ${ }^{14} \mathrm{CO}_{2}$ production rates were simultaneously simulated by taking the first derivative of cumulative ${ }^{14} \mathrm{CO}_{2}$ data generated.

Parameters were optimized via a three-step iPBPK-R framework (Fig. 3). In each step, a nested optimization was used. In the first and second steps, the fraction of the drug filtered via the glomerulus $(\delta)$ and the expression ratio of P-gp transporters between liver and kidney compartments were preestimated in the inner optimization loop of the iPBPK-R model fitting to the previously published mean ${ }^{14} \mathrm{CO}_{2}$ production rate at 20 minutes after ERMBT (Frassetto et al., 2007).

$1^{\text {st }}$ and $2^{\text {nd }}$ steps: PBPK modeling of ERMBT

(literature data of mean ${ }^{14} \mathrm{CO}_{2}$ production rate at 20 minutes)

Pre-estimation on P-gp parameters in a healthy population

Outer loop - IVIVE adjustment

- Maximum velocity of uptake transporter $\left(V_{\max , U P 1}\right)$

- CYP3A4 clearance ( $\left.\mathrm{CL}_{\mathrm{CYP}}\right)$

- Partition coefficient in the OT compartment $\left(\mathrm{P}_{\text {OT:P }}\right)$

- Exponential decay in the OT compartment $(\mu)$

Inner loop - model fitting

- Fraction of the drug filtered via the glomerulus $(\delta)$

- Distribution ratio of P-gp in the HC and KD compartments

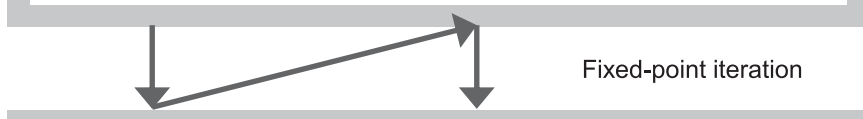

$3^{\text {rd }}$ step: Individualized PBPK modeling of ERMBT (iPBPK-R)

$\left({ }^{14} \mathrm{CO}_{2}\right.$ production rate data from 12 healthy subjects)

Nested 9-parameter estimation in healthy subjects

Outer loop - IVIVE adjustment

- $V_{\max , \text { UP1 }}$

- $\mathrm{CL}_{\mathrm{CYP}}$

- $\mathrm{P}_{\text {OT:P }}$

- $\mu$

- Maximum velocity of MRP2 $\left(V_{\max , \text { oUt1 }}\right)$

- Maximum velocity of P-gp $\left(V_{\max , \text { out2 } 2}\right)$

- Volume of hepatocyte $\left(V_{\mathrm{LC}}\right)$

- Blood flow into the OT compartment $\left(Q_{\mathrm{OT}}\right)$

- Total passive diffusion between ES and LC compartments $\left(Q_{E S-L C}\right)$

Inner loop - model fitting

- Scaling coefficient for $V_{\max }$ up 1

- Scaling coefficient for $V_{\max , \text { OUT1 }}$

- Scaling coefficient for $V_{\max \text {, OUT2 }}$

Fig. 3. Framework of the application of iPBPK-R to the ERMBT study in 12 healthy subjects.
In the same two steps, four PBPK parameters (maximum velocity of the meta-uptake transport process, CYP3A4 clearance, partition coefficient in the combined other organs, and exponential decay parameter $\mu$ ) were preestimated via the outer loop optimization. The preestimated parameter values in the first two steps were used as initial input values in the third step. In the third step (main step), the nested optimization process consisting of the inner loop and outer loop was used to optimize and estimated IVIVE adjustment factors for five PBPK parameters (maximum velocity of two efflux transporters P-gp and MRP2, volume of total hepatocyte, blood flow into the combined other organs, and total passive diffusion between extracellular space and hepatocyte). This was done so that we could implement iPBPK-R model fitting to the observed ${ }^{14} \mathrm{CO}_{2}$ production rates between 0 and 120 minutes in 12 healthy subjects. In this step, the model fit was evaluated using normalized residuals between predicted and observed rates, visual inspection, and plausibility of the resulting parameter estimates. Among the PBPK parameters in Table 1, nine outer loop parameters (see Fig. 1) were selected to optimize and estimate since they were essential parameters for model fitting based on visual investigation and sensitivity analysis. In fact, adjustment factors for maximum velocity of efflux transporters did not have a critical effect of controlling the shape of the ${ }^{14} \mathrm{CO}_{2}$ production rate-time curves. However, these parameters were kept for optimization since they were part of nonrenal elimination pathways. The three scaling parameters in the inner optimization loop in the third step (Fig. 3) were used to calculate final adjustment factors for IVIVE values of transporter kinetics. One cycle of fixed-point iteration was conducted and the results of the third step outer loop were used in subsequent simulations to refine the parameter estimates.

All of the simulations and parameter estimations were implemented with the programming language and free software environment R 3.4.4. The $\mathrm{R}$ package deSolve was used for solving the set of ODEs, and largescale simulations were conducted via the Bridges supercomputer at the Pittsburgh Computing Center (Towns et al., 2014). Methodological details of the iPBPK-R approach will be described in a methodological manuscript under preparation (Franchetti $\mathrm{Y}$ et al., manuscript in preparation). Some methodological features were discussed at the Society of Industrial and Applied Mathematics Conference on Computational Science and Engineering (Franchetti Y, Nolin TD, and Franchetti F. Towards precision medicine: Simulation based parameter estimation for drug metabolism. Society of Industrial and Applied Mathematics (SIAM) Conference on Computational Science and Engineering (CSE19), February 2019).

Statistical Analysis. Our primary aim was to simultaneously estimate pharmacokinetic (PK) parameters associated with metabolic versus transporter-mediated pathways within individual study subjects using iPBPK-R. Descriptive statistics were presented with the mean \pm S.D. and/or the median (range). The 25 th and 75 th percentiles were also obtained for creating box plots. This study explored feasibility and estimability on model parameters, and no statistical test was powered in advance. Assuming that all measurements were non-normally distributed, one-sided Wilcoxon-Mann-Whitney tests were conducted for comparing two independent groups. Spearman's $\rho$ correlation coefficients were calculated in correlation analysis for studying the relationships between estimated adjustment factors for nonrenal elimination parameters and baseline demographic or uremic solute concentrations [blood urea nitrogen (BUN), serum creatinine, and tumor necrosis factor- $\alpha$ ]. An adjustment factor of 1 means that there was no need to adjust the initial parameter input value in the optimization. A $P$ value $<0.05$ was considered statistically significant for all comparisons. All statistical analyses were conducted using SAS 9.4 (SAS Institute Inc., Cary, NC).

\section{Results}

The iPBPK-R model was applied to fit the observed ${ }^{14} \mathrm{CO}_{2}$ production rate data for the 12 healthy subjects (Fig. 4). The nested optimization procedure yielded parameter estimates 


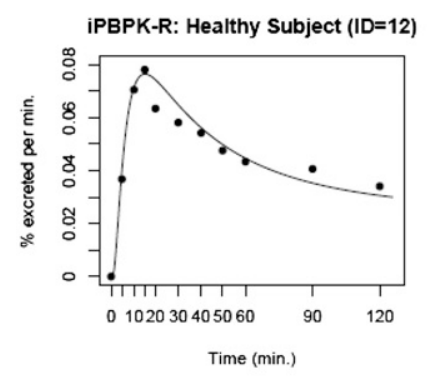

iPBPK-R: Healthy Subject (ID=18)

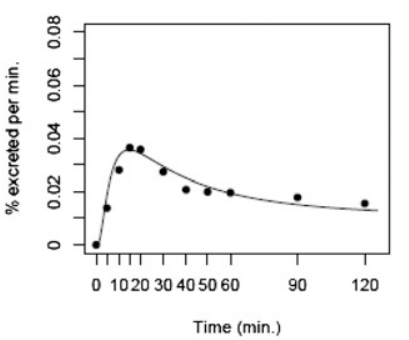

iPBPK-R: Healthy Subject (ID=22)

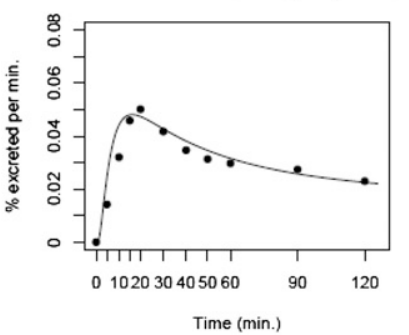

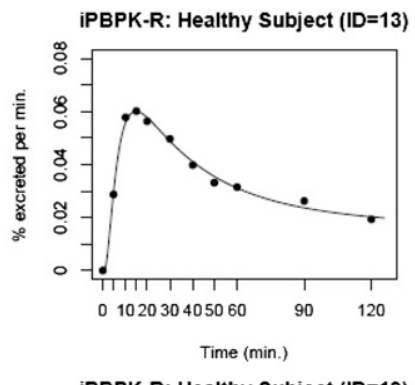

iPBPK-R: Healthy Subject $(I D=19)$

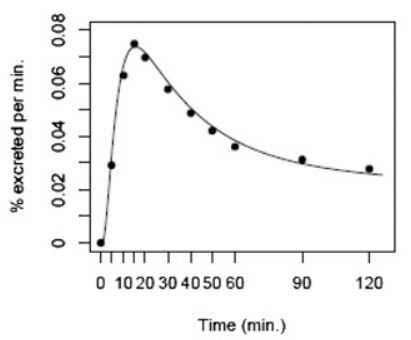

iPBPK-R: Healthy Subject (ID=23)

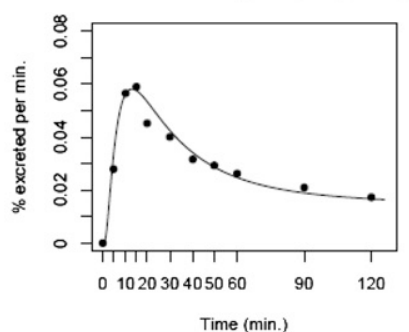

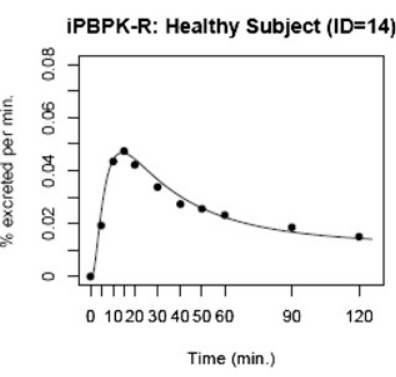

iPBPK-R: Healthy Subject (ID=20)

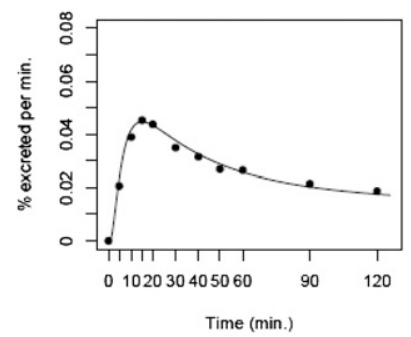

IPBPK-R: Healthy Subject (ID=24)

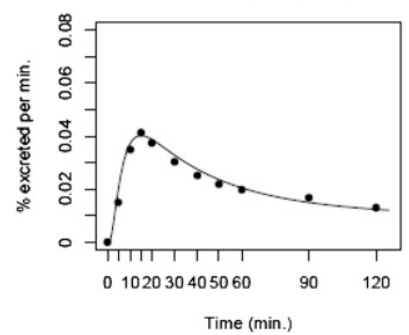

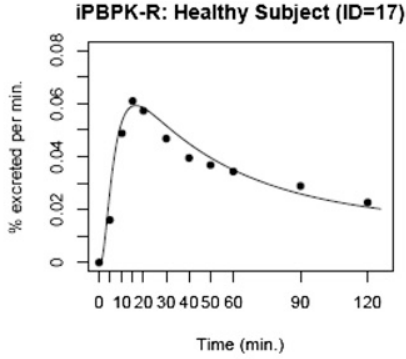

iPBPK-R: Healthy Subject $(I D=21)$

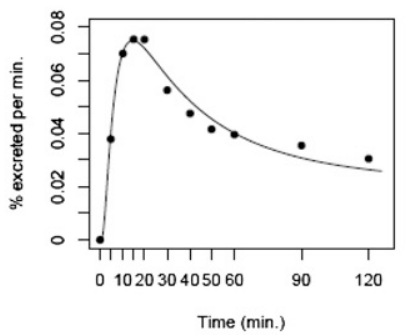

IPBPK-R: Healthy Subject (ID=25)

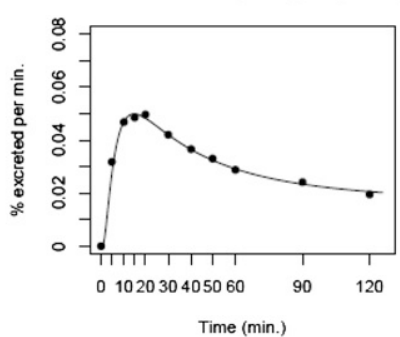

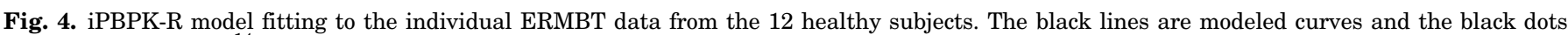
indicate the observed ${ }^{14} \mathrm{CO}_{2}$ production rates.

for all subjects, and the excellent model fit was confirmed with the normalized residual-time plots across subjects (mean $10^{-4}$, S.D. $10^{-4}$ ) (Fig. 5; Table 2; Supplemental Table 1).

The estimated adjustment factors for parameters in the liver and combined other organ compartments are shown in Figs. 6A and 7, respectively. The estimated median CYP3A4 clearance was $87.8 \%$ lower than the original IVIVE value (adjustment factor $=0.122$ in Fig. 6A) and the median adjustment factor for total passive diffusion was 33.5\% lower than the original IVIVE value (adjustment factor $=0.665$ in Fig. 6A). The adjustment factor for maximum velocity $\left(V_{\max }\right)$ of the meta-uptake transporter varied by individual (25th quantile adjustment factor, 0.74; 75th quantile adjustment factor, 0.99). When stratified by sex (Fig. 6B), the adjustment factor for $V_{\max }$ of the meta-uptake transporter did not differ by sex; a few female healthy subjects had reduced uptake transport activity (median adjustment factor in male/female subjects $=0.99$ : 0.67 ; one-sided exact $P=0.10)$. $V_{\max }$ of efflux transporters (P-gp and MRP2) did not require an adjustment from the original IVIVE values, since the estimated median and mean adjustment factors were 1.00. The median adjustment factors for total hepatocyte volume, CYP3A4 clearance, and total passive diffusion varied among individuals (Fig. 6A). In the combined other organs compartment (Fig. 7), arterial blood flow barely required IVIVE adjustment (median adjustment factor 0.87 or $13 \%$ decrease from the IVIVE value), whereas IVIVE values of exponential decay $\mu$ and partition coefficient needed to be adjusted as high as 4.0 and 18.8 times (by median), respectively. The latter two parameters did not vary by individual but the adjustment factor for arterial blood flow relatively varied by individual (25th quantile adjustment factor, $0.52 ; 75$ th quantile adjustment factor, 0.99). Since the meta-uptake transporter, CYP3A4 clearance, volume of total hepatocyte, and total passive diffusion varied in their adjustment factor, we stratified these adjustment factors by sex in the box-and-whisker plots (Fig. 6B). When compared by sex using one-sided Wilcoxon-Mann-Whitney tests, CYP3A4 clearance was significantly lower in male than in female subjects (median reduction from the IVIVE value in male/female subjects $=89.3 \%: 78.0 \%$; one-sided exact $P=0.04$ ). The total hepatocyte volume and total passive diffusion did not differ by sex (one-sided exact $P=0.27$ and 0.17 , respectively).

The observed ${ }^{14} \mathrm{CO}_{2}$ production rate-time curves of 12 healthy subjects had three phases: 1) the increasing absorption/distribution phase, 2) the first elimination phase, and 3 ) the second elimination phase (depicted by the black dots in Fig. 4). Through the sensitivity analysis in the iPBPK-R model fitting, we found that multiple physiologic parameters interact and control the shape of the ${ }^{14} \mathrm{CO}_{2}$ production ratetime curve (Fig. 8). When CYP3A4 clearance increases, the peak height and the area under the curve (AUC) increase with a steeper absorption phase. $V_{\max }$ of uptake drug transport and total passive diffusion together control the peak height, the first elimination phase, and the second elimination phase of the curve. As part of the sensitivity analysis, we simulated ${ }^{14} \mathrm{CO}_{2}$ production rate-time curves with one additional varying parameter (see three-dimensional plots in Fig. 9). 

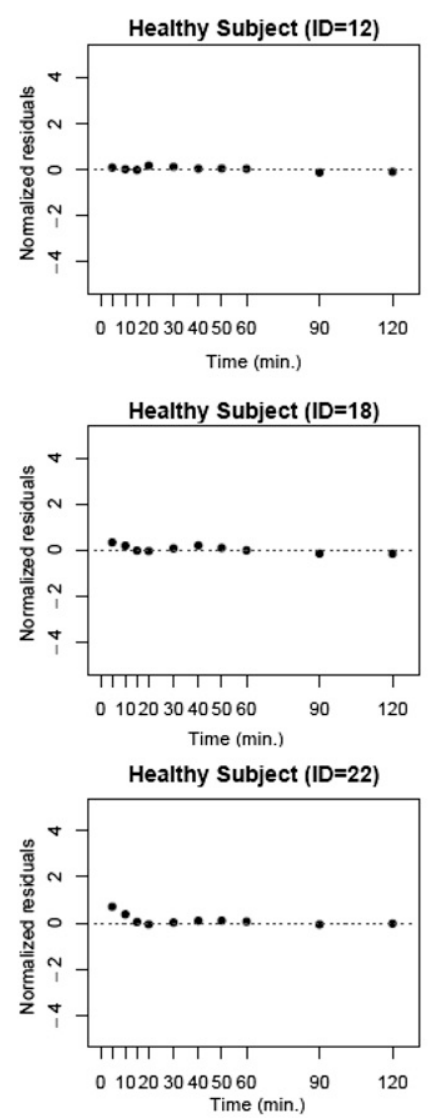
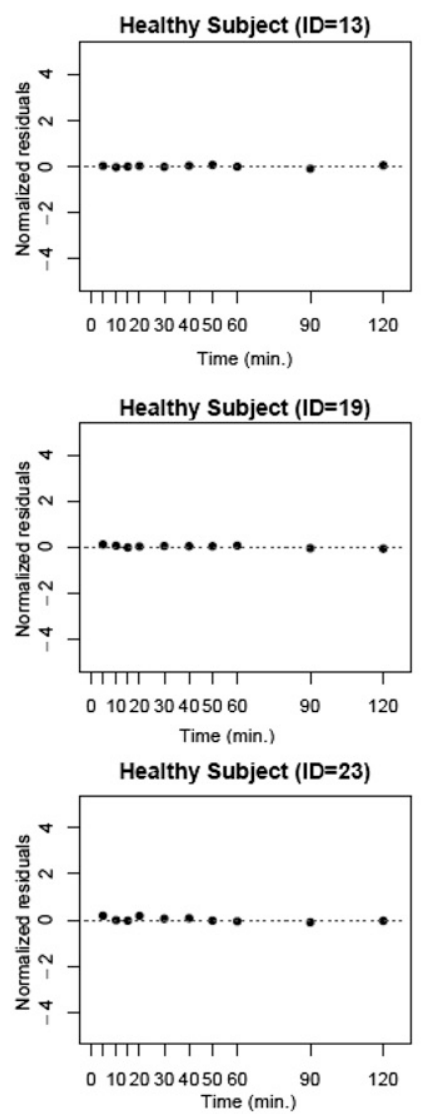
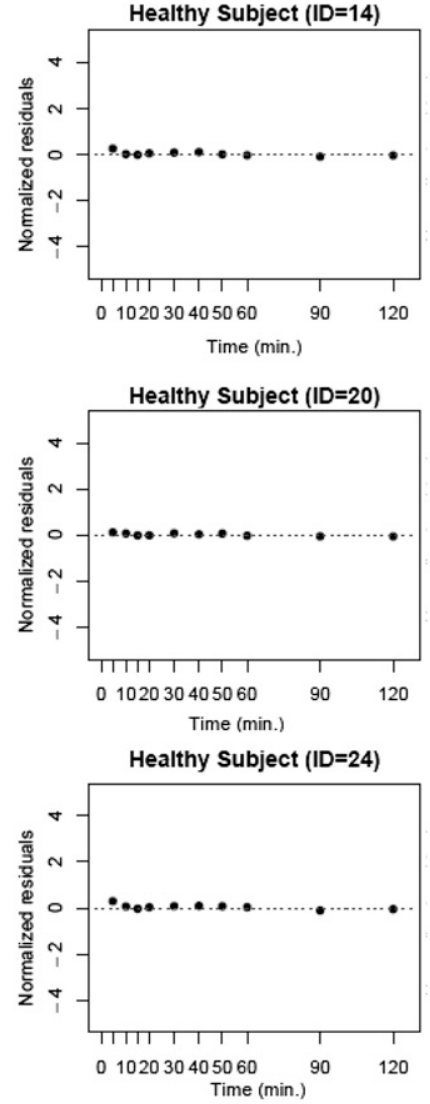
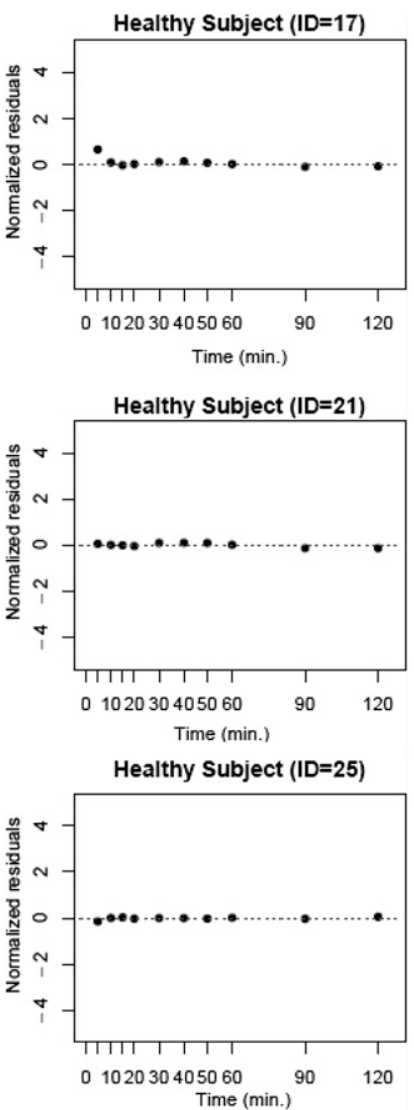

Fig. 5. Normalized residual-time plots for the 12 healthy subjects. Residuals were obtained from iPBPK-R model fitting to the ERMBT data (results are shown in Fig. 4). The black dots are normalized residuals.

In Fig. 9A, CYP3A4 clearance varied from $1 \%$ to $100 \%$ of the IVIVE value. In Fig. 9B, $V_{\max }$ of the meta-uptake transporter varied from $1 \%$ to $200 \%$ while keeping the total passive diffusion at $50 \%$ of its optimal value. Modeled ${ }^{14} \mathrm{CO}_{2}$ production rates were sensitive to changes in CYP3A4 clearance (see Fig. 9A). Changes in total passive diffusion and uptake drug transport together had an effect on the shape of ${ }^{14} \mathrm{CO}_{2}$ production rate-time curves (Fig. 9B).

As an exploratory analysis, Spearman correlation analysis between the estimated adjustment factors for nonrenal elimination parameters $\left(V_{\max }\right.$ of meta-uptake transporters, CYP3A4 clearance, and total passive diffusion) and baseline parameters was conducted (see Table 3; Supplemental Table 2). These baseline parameters were demographic and physical factors (height, weights, age, and estimated glomerular filtration rate) and uremic solute concentrations including serum creatinine, BUN (a low molecular weight solute), and tumor necrosis factor- $\alpha$ (a middle molecular weight solute). In all 12 subjects, the correlation coefficient between BUN and the adjustment factor for $V_{\max }$ of meta-uptake transporters was estimated to be negative and strong, and this correlation was statistically significant ( $\rho=-0.608 ; P=0.04$ ) (Table 3 ). In the male subjects, BUN was negatively correlated with the adjustment factors for $V_{\max }$ of meta-uptake transporters $(\rho=-0.857$; $P=0.01)$, CYP3A4 clearance $(\rho=-0.857 ; P=0.01)$, and passive diffusion $(\rho=-0.821 ; P=0.02)$, which were very strong according to Evans' correlation criteria (Evans, 1996) (Supplemental Table 2).

TABLE 2

Parameter estimates through the nested optimization and normalized residuals in the iPBPK-R modeling of the ERMBT study in 12 healthy subjects

\begin{tabular}{lcc}
\hline Estimated parameter & Mean \pm S.D. & Median (Range) \\
\hline IVIVE adjustment (adjustment factor) & & $0.98(0.23,1.00)$ \\
Meta-uptake transporter & $0.84 \pm 0.26$ & $1.00(1.00,1.00)$ \\
P-gp & $1.00 \pm 0.00$ & $1.00(1.00,1.00)$ \\
MRP2 & $1.00 \pm 0.00$ & $0.12(0.07,0.24)$ \\
CYP3A4 clearance & $0.14 \pm 0.06$ & $0.73(0.50,1.01)$ \\
Hepatocyte volume & $0.78 \pm 0.19$ & $18.78(18.72,18.84)$ \\
Partition coefficient in the combined other organs & $18.77 \pm 0.03$ & $0.87(0.45,1.22)$ \\
Blood flow in the combined other organs & $0.80 \pm 0.27$ & $3.99(3.97,4.03)$ \\
Exponential decay parameter in the combined other organs & $3.99 \pm 0.01$ & $0.67(0.13,0.87)$ \\
Total passive diffusion between ES and LC & $0.60 \pm 0.21$ & $0.0001\left(0,4 \times 10^{-4}\right)$ \\
Model fit: normalized residual & $10^{-4} \pm 10^{-4}$ & \\
\hline
\end{tabular}




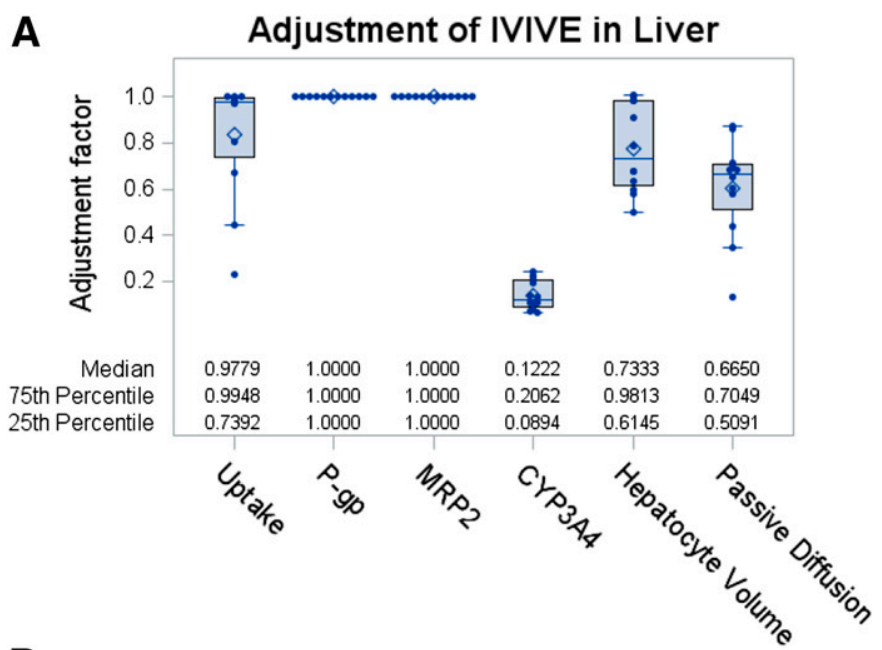

B

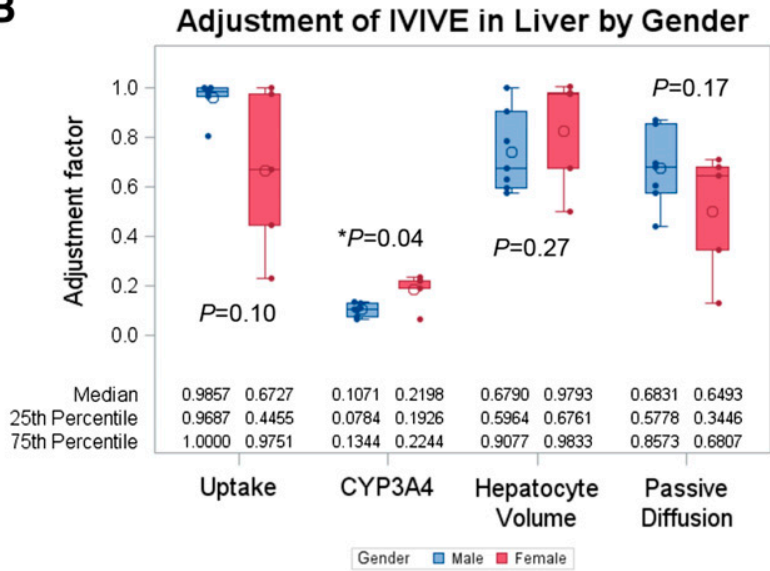

Fig. 6. (A) Estimated IVIVE adjustment factors for PBPK parameters in the liver compartments (ES and LC subcompartments). The PBPK parameters are maximum velocity of the meta-uptake transporter, maximum velocity of P-gp, maximum velocity of MRP2, CYP3A4 clearance, the volume of the hepatocyte, and total passive diffusion between ES and LC subcompartments (from the left to the right on the $x$-axis). These PBPK parameters were estimated in the main step (third step) of the iPBPK-R framework as shown in Fig. 3. (B) Estimated IVIVE adjustment factors in the liver compartments stratified by sex. These selected estimates adjust CYP3A4 clearance, the volume of the hepatocyte, and total passive diffusion between ES and LC subcompartments. When the IVIVE adjustment factor is estimated to be 1 , it is interpreted that the parameter input was not adjusted from the original IVIVE input value through the iPBPK-R model fitting. Exact $P$ values are shown based on Wilcoxon-Mann-Whitney tests for comparing between male and female subjects (one-sided test). $* P<0.05$ (statistically significant difference).

\section{Discussion}

This is the first study to show that the activity of multiple nonrenal elimination pathways can be estimated within individual study subjects with a single probe drug, erythromycin, via $\mathrm{PBPK}$ modeling and optimization-based model fitting of rate data (iPBPK-R). The iPBPK-R method is distinctly different from and advantageous over other approaches that use multiple pathway-specific probes (i.e., the "cocktail" approach). The cocktail approach may lead to higher imprecision due to 1) uncertainty in parameter estimates pertinent to different probes' characteristics, 2) varying interactions with physiologic properties, and 3) intercohort variabilities (Zhou et al., 2004; Smith et al., 2007). The iPBPK-R method overcomes these by utilizing the distinct and dynamic information contained in rate data solely obtained with a single probe.
Nonlinear model fitting of measured rate data (Oppenheim and Schafer, 2009) was adopted in our individualized PBPK modeling, which enabled multiple-parameter optimization to achieve high accuracy (see the normalized residual plots in Fig. 5). Figure 1 illustrates that the ${ }^{14} \mathrm{CO}_{2}$ production rate data contain more information than the cumulative ${ }^{14} \mathrm{CO}_{2}$ concentration data when they are observed. First, as shown in the inset panels for hypothetical subjects A, B, C, and D, the simulated curves of the cumulative amount of ${ }^{14} \mathrm{CO}_{2}$ generated during the study period are not sensitive to any underlying parameter changes since all four subjects' cumulative curves look similar. On the other hand, simulated curves of ${ }^{14} \mathrm{CO}_{2}$ production rates show a dynamic change depending on the parameter change, since the shape of the rate-time curves visibly differs across the subjects. This illustrates that, for a given measurement accuracy, measuring the first derivative data allows us to estimate parameter differences while these differences cannot be estimated from the original data. The iPBPK-R method used here leverages this for the purposes of characterizing multiple nonrenal clearance pathways simultaneously.

Historically, erythromycin was commonly used as a metabolic probe drug. It was administered intravenously and employed in the ERMBT to noninvasively measure in vivo activity of hepatic CYP3A4 (Turgeon et al., 1994; Hirth et al., 2000; Nolin et al., 2006a; de Wildt et al., 2007). However, it is now known that erythromycin undergoes hepatic clearance by OATPs, P-gp, and MRP2-mediated elimination pathways as well (Lan et al., 2000; Sun et al., 2004; Kurnik et al., 2006; Lam et al., 2006; Frassetto et al., 2007; Franke et al., 2011; Chu et al., 2013). Accordingly, interpretations of ERMBT data were misleading under the assumption that erythromycin clearance was dependent solely on CYP3A4mediated metabolism (Rivory and Watkins, 2001; Rivory et al., 2001). We were interested in accounting for the

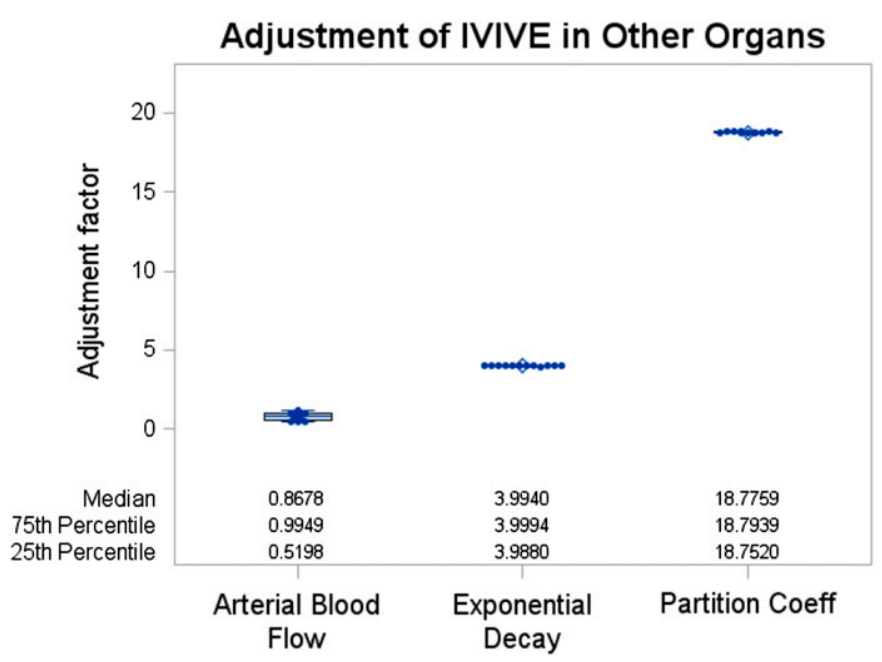

Fig. 7. Estimated IVIVE adjustment values for PBPK parameters in the combined other organs (OT) compartment. The PBPK parameters are arterial blood flow, exponential decay, and partition coefficient between OT and plasma, respectively (from the left to the right on the $x$-axis). These PBPK parameters were estimated in the main step (third step) of the iPBPK-R framework as shown in Fig. 3. When the IVIVE adjustment value is estimated to be 1 , it is interpreted that the parameter input was not adjusted from the original IVIVE input value through the iPBPK-R model fitting. 


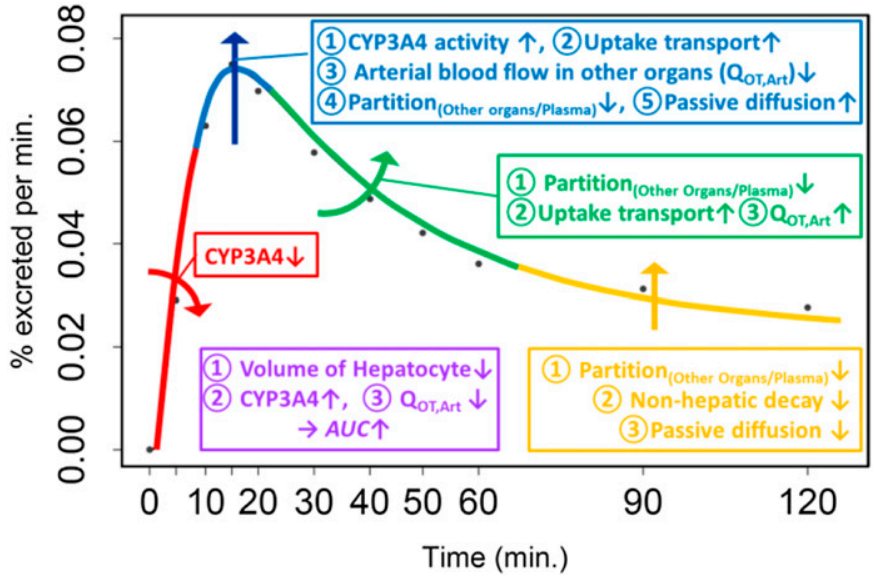

Fig. 8. ERMBT data interpretation as a result of the application of iPBPK-R. The modeled ${ }^{14} \mathrm{CO}_{2}$ concentration-time curve represents three phases: the increasing absorption phase, the first elimination phase after the peak of the curve, and the second elimination phase. Red line and text: The increased absorption phase gets less steep as CYP3A4 clearance decreases. Blue line and text: The peak of the curve increases as 1) CYP3A4 clearance increases, 2) maximum velocity of the meta-uptake transporter increases, 3 ) arterial blood flow in the combined other organ (OT) compartment (QоT,Art) decreases, 4) the partition coefficient of OT to plasma decreases, and/or 5) total passive diffusion between ES and LC subcompartments increases. Green line and text: the first elimination phase of the curve gets less steep as 1) the partition coefficient of OT to plasma decreases, 2) maximum velocity of the meta-uptake transporter increases, and/or 3) QoT,Art increases. Yellow line and text: the second elimination phase of the curve gets less steep or shifts upward as 1) the partition coefficient of OT to plasma decreases, 2) exponential decay in the OT compartment decreases, and/or 3) total passive diffusion between ES and LC subcompartments decreases. Purple text: the AUC of the curve increases as 1) the volume of the hepatocyte decreases, 2) CYP3A4 clearance increases, and/or 3) QoT,Art decreases.

multiple nonrenal elimination pathways of erythromycin and estimating corresponding kinetic parameters within individual healthy subjects through modeling observed rate data. Rate data is commonly used in engineering disciplines, typically in digital signal processing. We anticipated that by applying mechanistic iPBPK-R to the ERMBT data, we could simultaneously estimate the activity of multiple nonrenal elimination pathways. Although this is a pilot study, we found that CYP3A4 activity was consistently estimated to be lower than the IVIVE-based CYP3A4 activity in all subjects. We showed that CYP3A4 clearance, uptake transport, and other physiologic parameters collectively control the ${ }^{14} \mathrm{CO}_{2}$ production rates of the ERMBT (Figs. 8 and 9). This serves as a proof of principle that the iPBPK-R framework is a novel tool for delineating rate-limiting and nonrate-limiting elimination pathways using a single probe.

Simultaneous estimation of the activity of multiple nonrenal elimination pathways facilitates studying the interaction between enzymatic metabolism and drug transport or enzymetransporter interplay (Benet, 2009). As discussed previously, the ERMBT was actively used to probe in vivo CYP3A catalytic activity in the past. However, across clinical studies using the ERMBT, there were conflicting results in predicting total body clearance from the ${ }^{14} \mathrm{CO}_{2}$ production rate analysis. Rivory et al. (2001) discussed possible causes for the inconsistent results among the ERMBT studies. The reasons included high interindividual variability of the ${ }^{14} \mathrm{CO}_{2}$ production rate data, potential overestimation of ${ }^{14} \mathrm{CO}_{2}$ production in female subjects, and use of different type of measurements among studies
A Decrease in CYP clearance (\%)

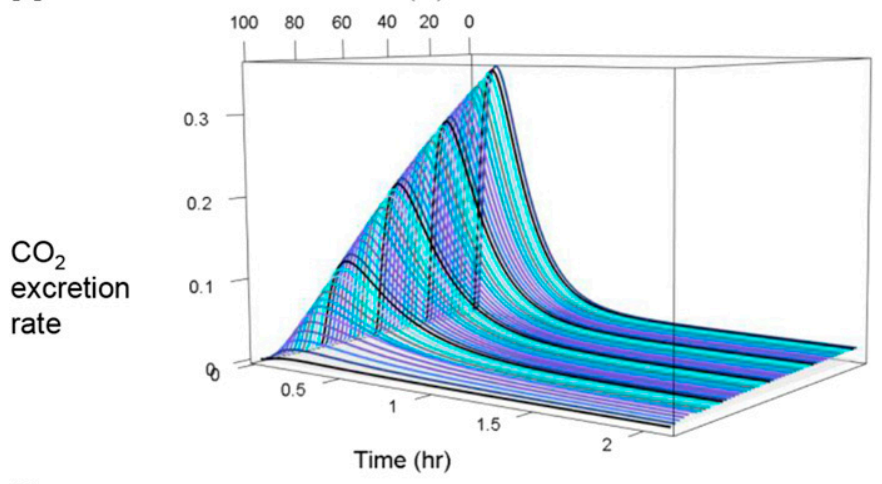

B

Increase in Uptake Maximum Velocity (\%)

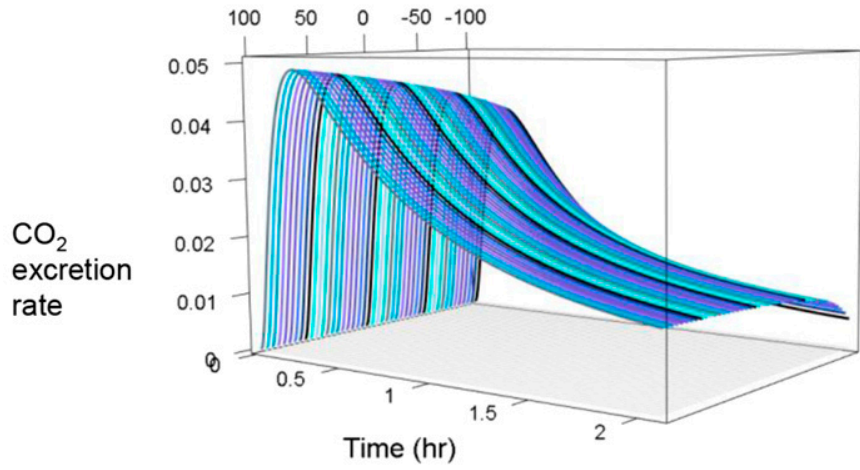

Fig. 9. Example of sensitivity analysis. Simulated $3 \mathrm{D}{ }^{14} \mathrm{CO}_{2}$ production rate-time curves where (A) CYP3A4 clearance was varied from $1 \%$ to $100 \%$ of the IVIVE value and (B) maximum velocity of the meta-uptake transporter was varied from $1 \%$ to $200 \%$ while keeping the total passive diffusion at $50 \%$ of its optimal value. CYP, cytochrome P450.

(i.e., inverse time to the peak, ${ }^{14} \mathrm{CO}_{2}$ production rate at 20 minutes, and AUC from 0 to 2 hours). Because erythromycin was found to be a substrate of not only CYP3A but also P-gp, Mrp2, and Oatp2 in in vitro studies (Sun et al., 2004; Franke et al., 2011), studying the effect of enzyme-transporter interplay on nonrenal drug elimination became essential (Chu et al., 2013). To study the enzyme-transporter interplay, mechanistic PBPK modeling is a useful tool because it allows us to explicitly describe the activity of each elimination pathway in the whole body system. By combining the set of mathematical equations with multiparameter optimization, one can indirectly estimate the activity of different elimination pathways in the target organ (Chu et al., 2013). The iPBPK-R approach overcomes infeasible direct sampling of drug concentrations from the target organ where the in vivo enzyme-transporter interplay may be occurring.

In studies using the ERMBT, ${ }^{14} \mathrm{CO}_{2}$ production may have been overestimated in female subjects and the difference between sexes was around 20\%-25\% (Rivory et al., 2001). We observed higher CYP3A4 activity in female than in male subjects and the difference between sexes was about $11 \%$. In fact, the result for female subjects aligned with the study of human liver bank samples in which expression levels of CYP3A4 in female samples were found to be higher than in male samples (Wolbold et al., 2003). No correlation was observed between CYP3A4 activity or drug transport activity and physiologic factors, weight, height, or body mass index in all subjects or in each sex. Accordingly, our study indicated that there is a sex-related difference in CYP3A4 activity. 
TABLE 3

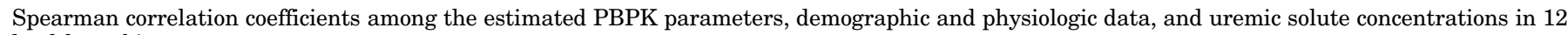
healthy subjects

\begin{tabular}{|c|c|c|c|c|c|c|c|c|}
\hline IVIVE adjustment (adjustment factor) & Height & Weight & BMI & Age & eGFR & $\mathrm{SCr}$ & BUN & TNF- $\alpha$ \\
\hline & $\mathrm{cm}$ & $k g$ & $\mathrm{~kg} / \mathrm{m}^{2}$ & years & $l / h$ & $m g / d l$ & $m g / d l$ & $\mathrm{pg} / \mathrm{ml}$ \\
\hline \multicolumn{9}{|l|}{ Meta-uptake transporter } \\
\hline$\rho^{a}$ & 0.371 & 0.147 & -0.259 & -0.372 & 0.007 & 0.351 & -0.608 & -0.161 \\
\hline$P$ value & 0.235 & 0.648 & 0.417 & 0.234 & 0.983 & 0.263 & $0.036^{b}$ & 0.618 \\
\hline \multicolumn{9}{|l|}{ CYP3A4 clearance } \\
\hline$\rho$ & -0.529 & -0.378 & 0.154 & 0.319 & -0.571 & 0.092 & -0.504 & -0.399 \\
\hline$P$ value & 0.077 & 0.225 & 0.633 & 0.312 & 0.053 & 0.775 & 0.095 & 0.199 \\
\hline \multicolumn{9}{|c|}{ Total passive diffusion between ES and LC } \\
\hline$\rho$ & 0.476 & 0.308 & -0.175 & -0.204 & -0.175 & 0.414 & -0.196 & 0.112 \\
\hline$P$ value & 0.117 & 0.330 & 0.589 & 0.526 & 0.586 & 0.181 & 0.542 & 0.729 \\
\hline
\end{tabular}

BMI, body mass index; eGFR, estimated glomerular filtration rate; SCr, serum creatinine; TNF- $\alpha$, tumor necrosis factor.- $\alpha$

${ }^{a}$ Spearman correlation coefficient.

${ }^{b} P<0.05$ indicates a statistically significant correlation.

We further conducted correlation analysis on the adjustment factors for nonrenal elimination clearance with plasma concentrations of uremic solutes. There was a significant inverse correlation between the uremic solute BUN and the adjustment factor for $V_{\max }$ of the meta-uptake transporter (OATPs) in 12 subjects $(\rho=-0.608)$. In male subjects, significant inverse correlations were observed between BUN and maximum velocity of OATPs, CYP3A4 clearance, and passive diffusion, respectively. Despite the small sample size, we showed that correlation analysis can be used to explore relationships between the activity of nonrenal elimination pathways and potential surrogate biomarkers (such as uremic solutes) after iPBPK-R modeling.

There are several limitations in this study. First, the detailed structures in the kidney compartment were omitted and were not modeled so that multiple parameters could be estimated using a reduced order model. Second, in the reduced order PBPK model, it is not possible to distinguish the value of maximum velocity and the number of individual transporters. Third, we did not distinguish in vitro parameter values between ${ }^{14} \mathrm{C}$-erythromycin and erythromycin for IVIVE calculations. The two compounds are not the same but they are used interchangeably in clinical research. Therefore, the literature values are the best parameters that we could use. Fourth, no genetic variant data of OATPs were available, so we could not conclude whether low CYP3A4 activity was truly caused by the sex difference or the interplay with OATP variants. Fifth, IVIVE values were calculated based on average $70-\mathrm{kg}$ male data. Although both male and female subjects were enrolled in the clinical study with ERMBT, the average body weight was close to $70 \mathrm{~kg}$ (68 kg). Therefore, use of the average 70-kg male data is not expected to cause a large bias. The variability in weight is reflected in the estimated adjustment factors for the total hepatic volume. Finally, the CYP3A4 and transporter activity estimates derived from iPBPK-R modeling were not compared with or validated using specific pharmacologic probe substrates as controls. The fitted model cannot be validated against the measurements beyond the breath data. However, the model is a reduced order model, and we could establish that the model was not overfitted. Furthermore, the visual inspection and normalized residuals calculated from predicted and observed curves indicated excellent model fit across all individual subjects. By conducting simultaneous optimizations of parameters and sensitivity analysis, we showed the robustness of parameter estimates and consistency in the estimated CYP3A4 activity across
12 subjects. Similarly, the estimated uptake transporter activity was robust and consistent across male subjects, whereas a wider variability was seen in female subjects.

iPBPK-R can be applied to any rate-derived data beyond ERMBT. Ideal characteristics of measurements are as follows: 1) rate measurement with long elimination half-life and frequent sampling, 2) in vitro data sufficient for IVIVE, 3 ) intravenous dosing, and 4) individual data. The underlying idea for measurement 1 is that rate information can be used to capture both non-rate-limiting and rate-limiting steps in elimination pathways of interest. As another example, breath rate data of volatile organic compounds can be used to study the role of potential biomarkers on hemodialysis efficiency (Grabowska-Polanowska et al., 2019). Other potential areas of application include drug-drug interaction, pathophysiological effects, and the effect of intervention, polypharmacy, or any other extrinsic factors on drug disposition by estimating adjustment factors and conducting correlation analysis. In the future, we plan to apply the iPBPK-R framework to rate data of other compounds that are known to be cleared by multiple nonrenal elimination pathways.

\section{Conclusion}

The iPBPK-R approach was applied to evaluate ${ }^{14} \mathrm{CO}_{2}$ production rate data of the ERMBT in healthy subjects. Optimized iPBPK-R models fit the individual data well and they allowed us to distinguish and simultaneously estimate the activity of multiple nonrenal elimination pathways of the single probe ${ }^{14} \mathrm{C}$-erythromycin. The median in vivo CYP3A4 activity was estimated as low as $12.2 \%$ of the IVIVE-based CYP3A4 clearance. ${ }^{14} \mathrm{CO}_{2}$ production rate data has rich information allowing estimation of per-person rate-limiting and nonrate-limiting PK parameters. Accordingly, we found that the multiple PK and physiologic parameters collectively control the ${ }^{14} \mathrm{CO}_{2}$ production rate-time curve of the ERMBT. Adjustment factors for the activity of drug transporters were also estimated in all subjects. Finally, correlation analysis can be used to explore relationships between the activity of nonrenal elimination pathways and potential surrogate biomarkers (such as uremic solutes) via iPBPK-R modeling. In summary, we applied iPBPK-R to ERMBT data to distinguish and simultaneously estimate the activity of multiple nonrenal elimination pathways in healthy subjects. This serves as proof of principle that the iPBPK-R framework is a novel tool for 
delineating rate-limiting and non-rate-limiting elimination pathways using a single probe.

\section{Acknowledgments}

The authors thank Professor Franz Franchetti (Department of Electrical and Computational Engineering, Carnegie Mellon University) for expertise, critical review, and insightful comments regarding the manuscript.

\section{Authorship Contributions}

Participated in research design: Franchetti, Nolin.

Conducted experiments: Franchetti.

Performed data analysis: Franchetti, Nolin.

Wrote or contributed to the writing of the manuscript: Franchetti, Nolin.

\section{References}

Araki H, Ogake N, Tsuneda R, Minami S, Watanabe Y, Tamai I, and Tsuji A (2002) Muscle distribution of antimicrobial agents after a single intravenous administration to rats. Drug Metab Pharmacokinet 17:237-244.

Barter ZE, Bayliss MK, Beaune PH, Boobis AR, Carlile DJ, Edwards RJ, Houston JB, Lake BG, Lipscomb JC, Pelkonen OR, et al. (2007) Scaling factors for the extrapolation of in vivo metabolic drug clearance from in vitro data: reaching a consensus on values of human microsomal protein and hepatocellularity per gram of liver. Curr Drug Metab 8:33-45.

Benet LZ (2009) The drug transporter-metabolism alliance: uncovering and defining the interplay. Mol Pharm 6:1631-1643.

Björkman S (2002) Prediction of the volume of distribution of a drug: which tissueplasma partition coefficients are needed? J Pharm Pharmacol 54:1237-1245.

Chu X, Korzekwa K, Elsby R, Fenner K, Galetin A, Lai Y, Matsson P, Moss A, Nagar S, Rosania GR, et al.; International Transporter Consortium (2013) Intracellular drug concentrations and transporters: measurement, modeling, and implications for the liver. Clin Pharmacol Ther 94:126-141.

de Wildt SN, Berns MJ, and van den Anker JN (2007) 13C-erythromycin breath test as a noninvasive measure of CYP3A activity in newborn infants: a pilot study. Ther Drug Monit 29:225-230.

Evans JD (1996) Straightforward Statistics for the Behavioral Sciences, Brooks/Cole, Belmont, CA.

Franke RM, Lancaster CS, Peer CJ, Gibson AA, Kosloske AM, Orwick S.J, Mathijssen RH, Figg WD, Baker SD, and Sparreboom A (2011) Effect of ABCC2 (MRP2) transport function on erythromycin metabolism. Clin Pharmacol Ther 89:693-701.

Frassetto LA, Poon S, Tsourounis C, Valera C, and Benet LZ (2007) Effects of uptake and efflux transporter inhibition on erythromycin breath test results. Clin Pharmacol Ther 81:828-832.

Freitas RA (1999) Nanomedicine Volume 1: Basic Capabilities, Landes Bioscience, Georgetown, TX.

Gaohua L, Wedagedera J, Small BG, Almond L, Romero K, Hermann D, Hanna D, Jamei M, and Gardner I (2015) Development of a multicompartment permeabilitylimited lung PBPK model and its application in predicting pulmonary pharmacokinetics of antituberculosis drugs. CPT Pharmacometrics Syst Pharmacol 4:605-613.

Grabowska-Polanowska B, Miarka P, Skowron M, Chmiel G, Pietrzycka A, and Śliwka I (2019) Breath analysis as promising indicator of hemodialysis efficiency. Clin Exp Nephrol 23:251-257.

Grillo JA, Zhao P, Bullock J, Booth BP, Lu M, Robie-Suh K, Berglund EG, Pang KS, Rahman A, Zhang L, et al. (2012) Utility of a physiologically-based pharmacokinetic (PBPK) modeling approach to quantitatively predict a complex drug-drugdisease interaction scenario for rivaroxaban during the drug review process: implications for clinical practice. Biopharm Drug Dispos 33:99-110.

Heikkinen AT, Parrott N, Dunkley T, and Cutler P (2016) Targeted proteomics to support transporter IVIVE and PBPK, in Drug Transporters: Volume 2: Recent Advances and Emerging Technologies (Nicholls G and Youdim K eds) pp 44-72, Royal Society of Chemistry, London.

Hirth J, Watkins PB, Strawderman M, Schott A, Bruno R, and Baker LH (2000) The effect of an individual's cytochrome CYP3A4 activity on docetaxel clearance. Clin Cancer Res 6:1255-1258.

Jamei M, Bajot F, Neuhoff S, Barter Z, Yang J, Rostami-Hodjegan A, and RowlandYeo K (2014) A mechanistic framework for in vitro-in vivo extrapolation of liver membrane transporters: prediction of drug-drug interaction between rosuvastatin and cyclosporine. Clin Pharmacokinet 53:73-87.

Jansson R, Bredberg U, and Ashton M (2008) Prediction of drug tissue to plasma concentration ratios using a measured volume of distribution in combination with lipophilicity. J Pharm Sci 97:2324-2339.

Kanamitsu S, Ito K, Green CE, Tyson CA, Shimada N, and Sugiyama Y (2000) Prediction of in vivo interaction between triazolam and erythromycin based on in vitro studies using human liver microsomes and recombinant human CYP3A4. Pharm Res 17:419-426.

Khor SP and Mayersohn M (1991) Potential error in the measurement of tissue to blood distribution coefficients in physiological pharmacokinetic modeling. Residual tissue blood. I. Theoretical considerations. Drug Metab Dispos 19:478-485.
Krecic-Shepard ME, Barnas CR, Slimko J, Gorski JC, Wainer IW, and Schwartz JB (1999) In vivo comparison of putative probes of CYP3A4/5 activity: erythromycin, dextromethorphan, and verapamil. Clin Pharmacol Ther 66:40-50.

Krivoruk Y, Kinirons MT, Wood AJ, and Wood M (1994) Metabolism of cytochrome $\mathrm{P} 4503 \mathrm{~A}$ substrates in vivo administered by the same route: lack of correlation between alfentanil clearance and erythromycin breath test. Clin Pharmacol Ther 56:608-614.

Kurnik D, Wood AJ, and Wilkinson GR (2006) The erythromycin breath test reflects P-glycoprotein function independently of cytochrome P450 3A activity. Clin Pharmacol Ther 80:228-234.

Lam JL, Okochi H, Huang Y, and Benet LZ (2006) In vitro and in vivo correlation of hepatic transporter effects on erythromycin metabolism: characterizing the importance of transporter-enzyme interplay. Drug Metab Dispos 34:1336-1344.

Lan LB, Dalton JT, and Schuetz EG (2000) Mdr1 limits CYP3A metabolism in vivo. Mol Pharmacol 58:863-869.

Langer O, Karch R, Müller U, Dobrozemsky G, Abrahim A, Zeitlinger M, Lackner E, Joukhadar C, Dudczak R, Kletter K, et al. (2005) Combined PET and microdialysis for in vivo assessment of intracellular drug pharmacokinetics in humans. J Nucl Med 46:1835-1841.

Levitt DG (2003) The pharmacokinetics of the interstitial space in humans. BMC Clin Pharmacol 3:3.

Maeda K and Sugiyama Y (2013) Prediction of hepatic transporter-mediated drug-drug interaction from in vitro data, in Transporters in Drug Development: Discovery, Optimization, Clinical Study and Regulation (Sugiyama Y and Steffansen B eds) pp 121-153, Springer, New York.

Nolin TD (2008) Altered nonrenal drug clearance in ESRD. Curr Opin Nephrol Hypertens 17:555-559.

Nolin TD, Appiah K, Kendrick SA, Le P, McMonagle E, and Himmelfarb J (2006a) Hemodialysis acutely improves hepatic CYP3A4 metabolic activity. $J$ Am Soc Nephrol 17:2363-2367.

Nolin TD, Appiah K, Kendrick SA, Phuong Le McMonagle E, and Himmelfarb J (2006b) Effect of hemodialysis on hepatic CYP3A4 activity (Abstract PI-60). Clin Pharmacol Ther 79:P23.

O'Hara ME, Clutton-Brock TH, Green S, and Mayhew CA (2009) Endogenous volatile organic compounds in breath and blood of healthy volunteers: examining breath analysis as a surrogate for blood measurements. J Breath Res 3:27005.

Oppenheim AV and Schafer RW (2009) Discrete-Time Signal Processing, 3rd ed, Prentice Hall, Upper Saddle River, NJ.

Paine SW, Parker AJ, Gardiner P, Webborn PJ, and Riley RJ (2008) Prediction of the pharmacokinetics of atorvastatin, cerivastatin, and indomethacin using kinetic models applied to isolated rat hepatocytes. Drug Metab Dispos 36:1365-1374.

Rivory LP, Slaviero KA, Hoskins JM, and Clarke SJ (2001) The erythromycin breath test for the prediction of drug clearance. Clin Pharmacokinet 40:151-158.

Rivory LP and Watkins PB (2001) Erythromycin breath test. Clin Pharmacol Ther 70:395-399.

Rowland M and Tozer TN (2005) Clinical Pharmacokinetics and Pharmacodynamics, Lippincott Williams and Wilkins, Philadelphia.

Schilders WH, Van der Vorst HA, and Rommes J (2008) Model Order Reduction Theory, Research Aspects and Applications, Springer, Berlin.

Smith NF, Raynaud FI, and Workman P (2007) The application of cassette dosing for pharmacokinetic screening in small-molecule cancer drug discovery. Mol Cancer Ther 6:428-440.

Spivey A (2000) Safer doses are a breath away, Endeavors, Office of the Vice Chancellor for Research at the University of North Carolina at Chapel Hill, Chapel Hill, NC.

Sugiyama E, Kikuchi A, Inada M, and Sato H (2011) The use of 13C-erythromycin as an in vivo probe to evaluate CYP3A-mediated drug interactions in rats. $J$ Pharm Sci 100:3995-4005.

Sun H, Frassetto LA, Huang Y, and Benet LZ (2010) Hepatic clearance, but not gut availability, of erythromycin is altered in patients with end-stage renal disease. Clin Pharmacol Ther 87:465-472.

Sun H, Huang Y, Frassetto L, and Benet LZ (2004) Effects of uremic toxins on hepatic uptake and metabolism of erythromycin. Drug Metab Dispos 32:1239-1246.

Towns J, Cockerill T, Dahan M, Foster I, Gaither K, Grimshaw A, Hazlewood V, Lathrop S, Lifka D, and Peterson GD (2014) XSEDE: accelerating scientific discovery. Comput Sci Eng 16:62-74.

Turgeon DK, Leichtman AB, Blake DS, Schmouder RL, Lown KS, Annesley TM, and Watkins PB (1994) Prediction of interpatient and intrapatient variation in OG 37-325 dosing requirements by the erythromycin breath test. A prospective study in renal transplant recipients. Transplantation 57:1736-1741.

Watkins PB (1994) Noninvasive tests of CYP3A enzymes. Pharmacogenetics 4: $171-184$

Wolbold R, Klein K, Burk O, Nüssler AK, Neuhaus P, Eichelbaum M, Schwab M, and Zanger UM (2003) Sex is a major determinant of CYP3A4 expression in human liver. Hepatology 38:978-988.

Yabe Y, Galetin A, and Houston JB (2011) Kinetic characterization of rat hepatic uptake of 16 actively transported drugs. Drug Metab Dispos 39:1808-1814.

Zhou H, Tong Z, and McLeod JF (2004) "Cocktail" approaches and strategies in drug development: valuable tool or flawed science? J Clin Pharmacol 44:120-134.

Address correspondence to: Dr. Thomas D. Nolin, Department of Pharmacy and Therapeutics, Center for Clinical Pharmaceutical Sciences, University of Pittsburgh School of Pharmacy, 208 Salk Pavilion, 335 Sutherland Dr., Pittsburgh, PA 15261. E-mail: nolin@pitt.edu 